\title{
Harnessing Sulfinyl Nitrenes: A Unified One-Pot Synthesis of Sulfoximines and Sulfonimidamides
}

Thomas Q. Davies, Michael J. Tilby, Jack Ren, Nicholas A. Parker, David Skolc, Adrian Hall, Fernanda Duarte, and Michael C. Willis*

Cite This: J. Am. Chem. Soc. 2020, 142, 15445-15453

Read Online

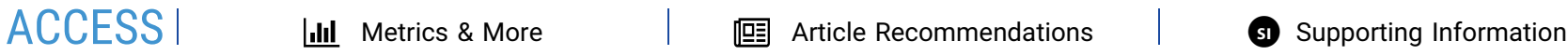

ABSTRACT: Sulfoximines and sulfonimidamides are promising compounds for medicinal and agrochemistry. As monoaza analogues of sulfones and sulfonamides, respectively, they combine good physicochemical properties, high stability, and the ability to build complexity from a three-dimensional core. However, a lack of quick and efficient methods to prepare these compounds has hindered their uptake in molecule discovery programmes. Herein, we describe a unified, one-pot approach to both sulfoximines and sulfonimidamides, which exploits the high electrophilicity of sulfinyl nitrenes. We generate these rare reactive intermediates

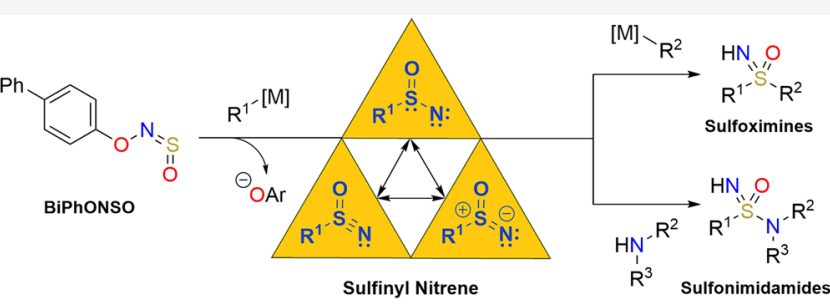

15 minute synthesis • alkyl, (hetero)aryl, alkenyl substituents • >70 examples stable, solid reagent $\cdot$ medicinally relevant products $\cdot$ DFT mechanistic studies from a novel sulfinylhydroxylamine $(\mathrm{R}-\mathrm{O}-\mathrm{N}=\mathrm{S}=\mathrm{O})$ reagent through an $\mathrm{N}-\mathrm{O}$ bond fragmentation process. Combining sulfinyl nitrenes with carbon and nitrogen nucleophiles enables the synthesis of sulfoximines and sulfonimidamides in a reaction time of just $15 \mathrm{~min}$. Alkyl, (hetero)aryl, and alkenyl organometallic reagents can all be used as the first or second component in the reaction, while primary and secondary amines, and anilines, all react with high efficiency as the second nucleophile. The tolerance of the reaction to steric and electronic factors has allowed for the synthesis of the most diverse set of sulfoximines and sulfonimidamides yet described. Experimental and computational investigations support the intermediacy of sulfinyl nitrenes, with nitrene formation proceeding via a transient triplet intermediate before reaching a planar singlet species.

\section{INTRODUCTION}

Sulfur(VI) compounds have played an outsized role in the development of medicines. Sulfones and sulfonamides in particular are key components of numerous drugs as well as agrochemicals. ${ }^{1}$ Replacing one of the oxygen atoms in these molecules with a nitrogen atom gives sulfoximines ${ }^{2}$ and sulfonimidamides. ${ }^{3}$ These compounds have recently gained traction in medicinal chemistry, first as isosteric replacements for their oxygenated analogues, ${ }^{4}$ and more recently due to increasing recognition of their potent mix of physicochemical properties. Being inherently chiral at sulfur, polar, and threedimensional, with good aqueous solubility and high chemical and metabolic stability, ${ }^{5}$ sulfoximines and sulfonimidamides represent an invaluable addition to the medicinal chemist's toolbox (Figure 1). ${ }^{6}$ However, although sulfonimidamides are increasingly common in pharmaceutical patents, ${ }^{7}$ and while sulfoximines appear in several clinical candidates ${ }^{8}$ and have been incorporated into a marketed agrochemical, ${ }^{9}$ they arguably still lack widespread recognition and are not yet routinely used in drug discovery. A significant reason for this is the inability to prepare these molecules quickly, ideally in one step, from widely available precursors. Considerable advances have been made in recent years in the synthesis of sulfoximines and sulfonimidamides by the Bolm and Bull groups, as well as

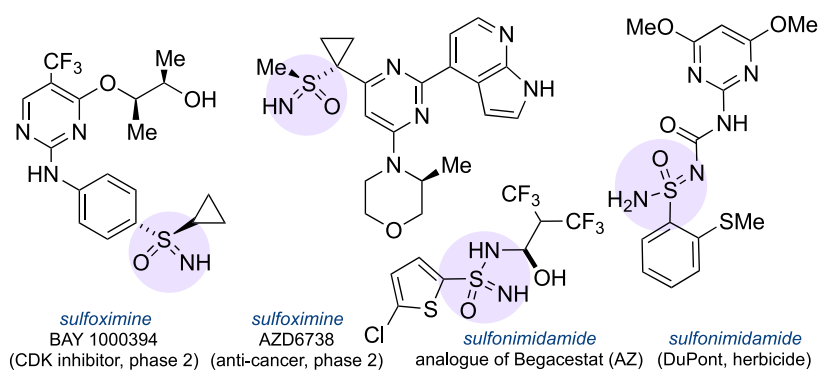

Figure 1. Bioactive aza-S(VI)-derivatives.

others, which have simplified the imination of thioethers, ${ }^{10}$ sulfoxides, ${ }^{11}$ and sulfenamides. ${ }^{12}$ Sulfonimidates ${ }^{13}$ and sulfinamides ${ }^{14}$ are also established as useful intermediates toward these targets. However, these methods share the fundamental

Received: June 29, 2020

Published: August 25, 2020 
limitation of ultimately needing thiol starting materials, which can be unpleasant to use due to their odor, oxidize to form disulfides in air, and are not widely commercially available. Consequently, the preparation of sulfoximines and sulfonimidamides often requires a multistep synthetic campaign featuring multiple oxidations, and in the case of sulfonimidamides typically involving moisture-sensitive intermediates such as sulfinyl chlorides. $5,15,16$

A synthetic approach starting from common nucleophiles and a central sulfur-containing electrophilic reagent would allow for more convenient access to a variety of sulfur(VI) derivatives. One embodiment of such a strategy is the recently reported SuFEx chemistry ${ }^{17}$ that uses iminosulfur oxidifluorides $\left(\mathrm{RN}=\mathrm{S}(\mathrm{O}) \mathrm{F}_{2}\right)$ as the electrophiles, in combination with a variety of heteroatom and carbon nucleophiles. ${ }^{18}$ In these examples, the key iminosulfur oxidifluorides are prepared from $\mathrm{SOF}_{4}$ gas, which is in turn prepared from gaseous $\mathrm{SF}_{4}$. In an alternative approach, we reported a one-pot, multistep, synthesis of sulfonimidamides from the sulfinylamine reagent $\mathrm{N}$-sulfinyltritylamine (TrNSO), organometallic reagents, and amines, albeit with the requirement of an intermediate oxidative step (Scheme 1, eq 1). ${ }^{19,20}$ The scope of this reaction proved to be broad, and it has now found use in

Scheme 1. Sulfinylamine Reagents in the Synthesis of Sulfonimidamides and Sulfondiimines, Both Featuring $\mathrm{S}(\mathrm{IV})$ to $\mathrm{S}(\mathrm{VI})$ Oxidations

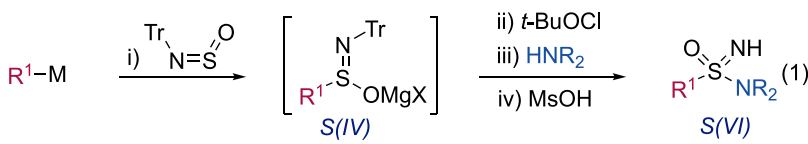

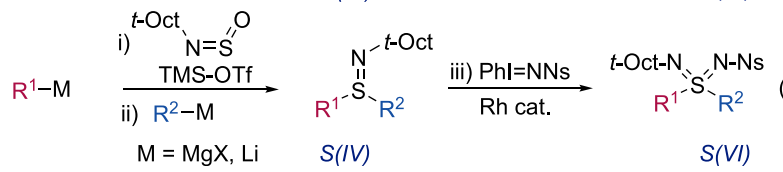

pharmaceutical companies, ${ }^{21}$ leading to TrNSO being commercially available. More recently we described a second sulfinylamine reagent, this time featuring a tert-alkyl substituent ( $t$-Oct-NSO), and used this reagent to prepare sulfilimines, en route to sulfondiimines. ${ }^{22}$ As with the TrNSO chemistry, this route to sulfondiimines again required a $S(I V)$ to $S(V I)$ oxidation using an external oxidant (Scheme 1, eq 2). Neither of these sulfinylamine reagents allowed the direct synthesis of sulfoximines from an electrophilic S(VI)-intermediate. This is a common shortcoming of many sulfur(VI) electrophiles including sulfonimidoyl chlorides, ${ }^{15}$ fluorides, ${ }^{23}$ and esters, ${ }^{24}$ which tend to undergo reduction, or show low reactivity, when combined with carbon nucleophiles. In particular, sulfonimidoyl fluorides must be combined with highly basic organolithium reagents, as they provide a mixture of reduction and substitution with less reactive Grignard reagents. ${ }^{25}$ Meanwhile, sulfonimidate esters generally require an excess ( 2 or more equivalents) of the organomagnesium reagent to be used. ${ }^{13 \mathrm{~b}}$

In our search for a general solution to this problem we were intrigued by the anomalous properties of little-known sulfinyl nitrenes. While sulfonyl nitrenes are among the most widely employed in modern nitrene chemistry, it is striking that the related species, sulfinyl nitrenes, which contain just one $\mathrm{S}=\mathrm{O}$ bond instead of two, are almost completely absent from the literature $^{26}$ since their initial report over 40 years ago by Maricich. ${ }^{27}$ Sulfonyl nitrenes undergo classic $\mathrm{C}-\mathrm{H}$ insertion and aziridination reactions via the nitrogen atom. In contrast,
Scheme 2. (a) Sulfonyl Nitrene versus Sulfinyl Nitrene Reactivity; (b) Sulfinyl Nitrene Resonance Forms
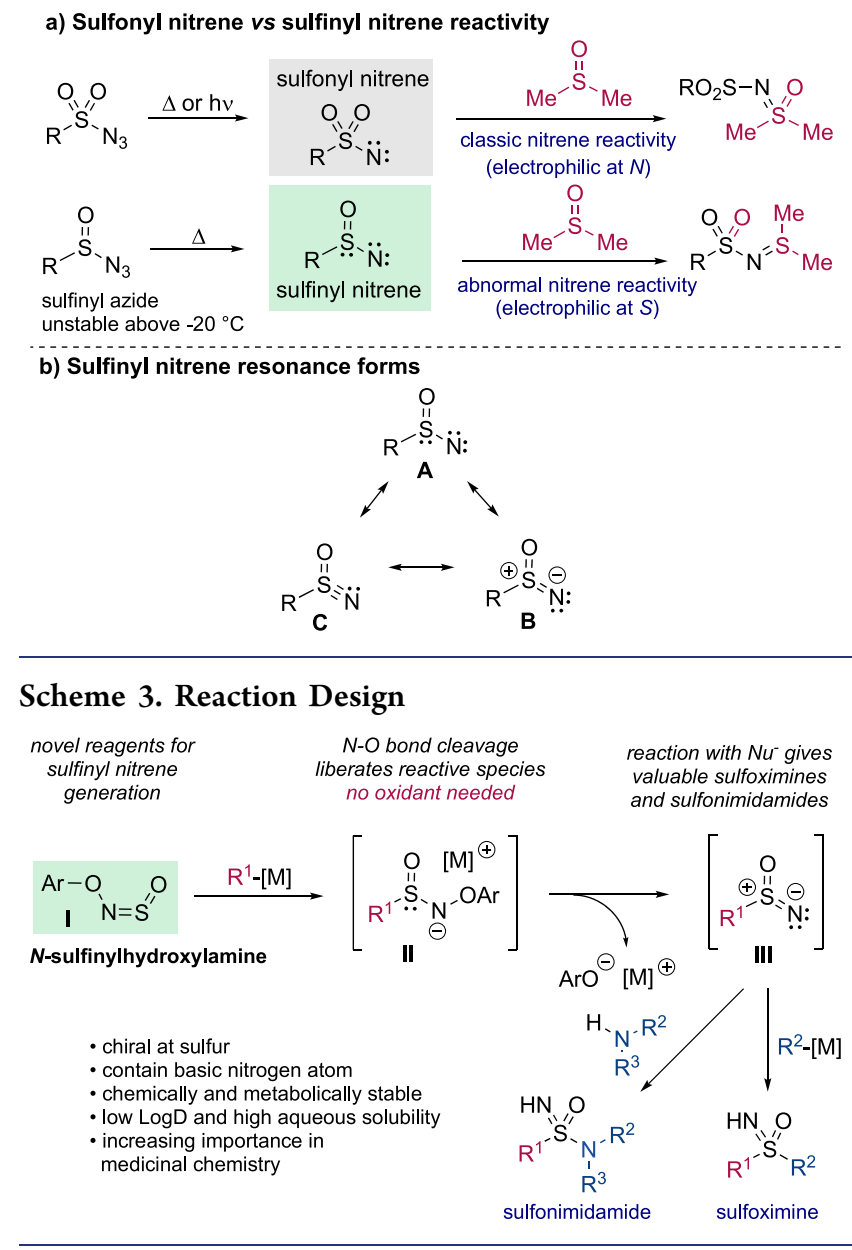

the known reactions of sulfinyl nitrenes proceed by electrophilic attack at sulfur. For example, sulfonyl nitrenes react with sulfoxides to give sulfoximines via attack of the sulfur lone pair of the sulfoxide onto the nitrogen atom of the nitrene. Sulfinyl

Scheme 4. BiPhONSO and Initial Reactions

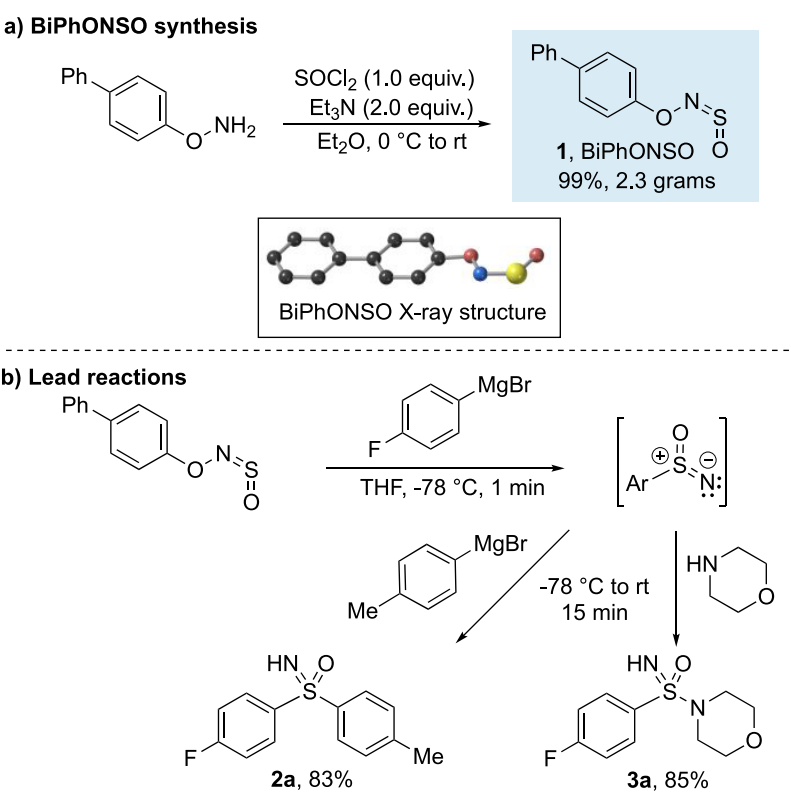


Table 1. (a) Scope of Sulfoximine Synthesis; (b) Four-Component Sulfoximine Synthesis

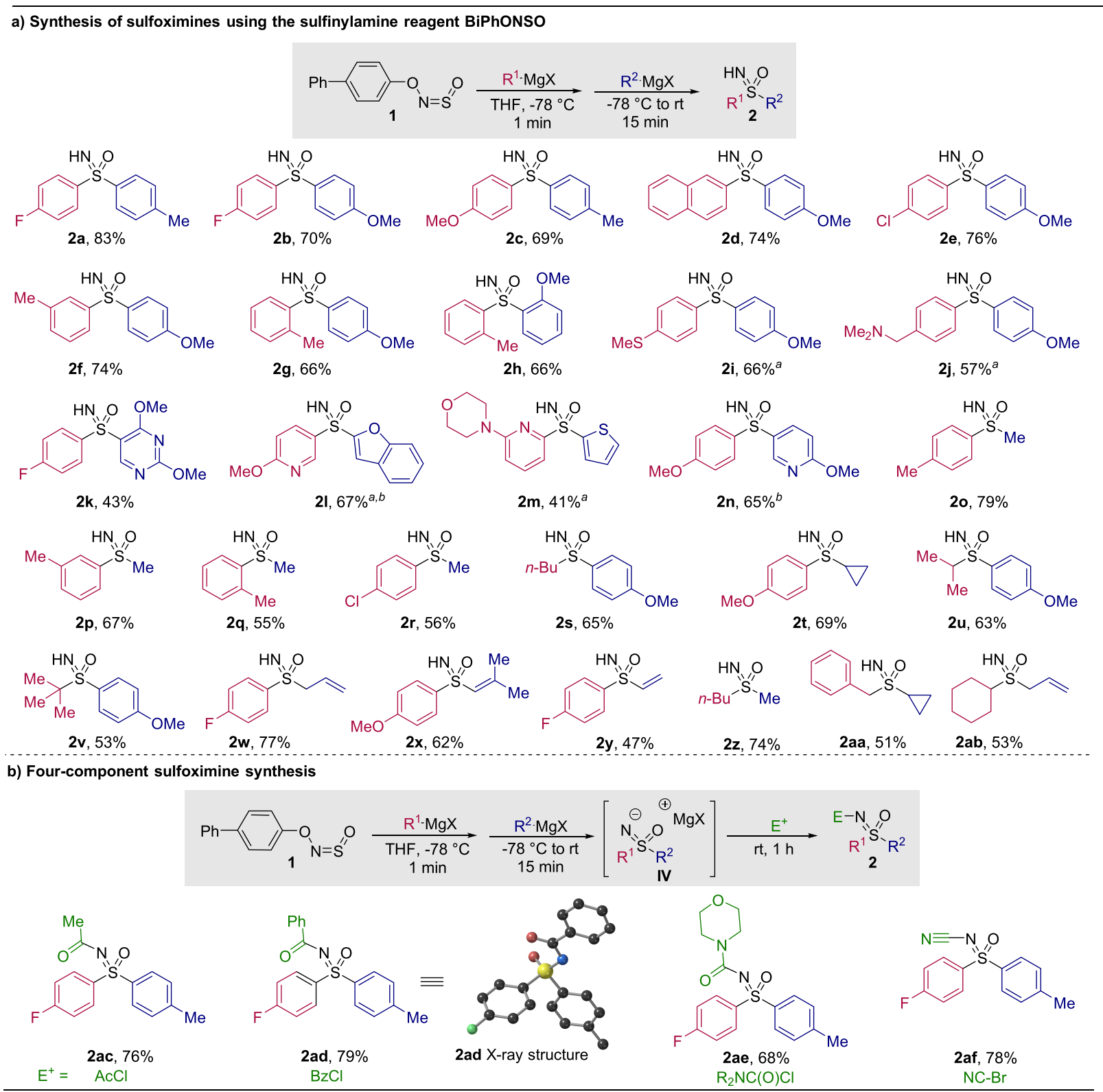

${ }^{a}$ Organolithium reagent used as 1 st organometallic reagent. ${ }^{b}$ Organolithium reagent used as 2 nd organometallic reagent.

nitrenes, however, react with sulfoxides by attack of the sulfoxide oxygen at the sulfur atom of this nitrene, and after rearrangement provide $N$-sulfonyl sulfilimines (Scheme $2 \mathrm{a}){ }^{27 \mathrm{~b}}$ This stark divergence in reactivity can be rationalized by considering the resonance structures of sulfinyl nitrenes (AB-C, Scheme 2b); the zwitterionic species $\mathbf{B}$, bearing a formal positive charge on sulfur and negative charge on nitrogen, gives a representative picture of sulfinyl nitrene reactivity. Because of these anomalous properties, we speculated that these species had the potential to transform the synthesis of sulfoximines and sulfonimidamides. Nevertheless, sulfinyl nitrenes remain underexplored and have received only minimal attention. ${ }^{28}$ This is mainly due to their known precursors, sulfinyl azides, being noted to be unstable above $-20{ }^{\circ} \mathrm{C}$ and exploding upon warming to room temperature, as well as delivering irreproducible results. tert-Butyl sulfinyl azide has been generated and decomposed in situ in the presence of water to provide the corresponding sulfonamide. ${ }^{26}$ However, attempts to employ amine or thiol nucleophiles with sulfinyl azides resulted only in substitution of the azide group. ${ }^{29}$ Given these challenges associated with the use of sulfinyl azides, we postulated that if we could identify a safe and convenient method to generate sulfinyl nitrenes, then new routes to a range of $S(\mathrm{VI})$ derivatives should be possible. Herein, we report such a system and show that by exploiting sulfinyl nitrene intermediates we can achieve efficient routes to both sulfoximines and sulfonimidamides.

\section{RESULTS AND DISCUSSION}

We envisioned that a sulfinylamine reagent bearing an appropriate leaving group on nitrogen would, when reacted with a carbon nucleophile, give convenient access to sulfinyl nitrenes. Our reaction design therefore centered on the use of a novel sulfinylhydroxylamine ${ }^{19,22,30}$ (I, Scheme 3). Upon 
Table 2. Scope of Sulfonimidamide Synthesis Exploiting the BiPhONSO Reagent

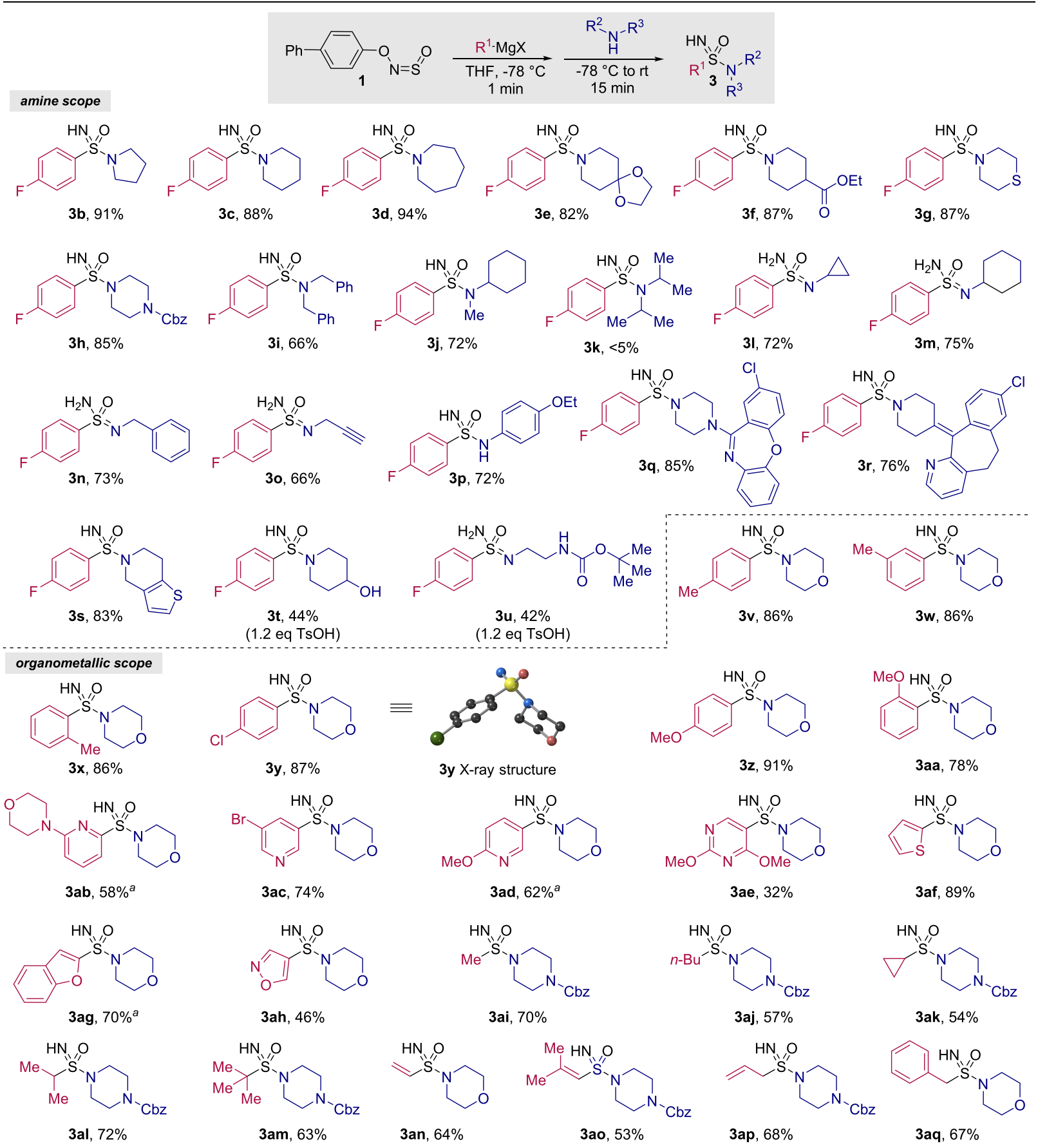

${ }^{a}$ Organolithium reagent used in place of Grignard reagent.

combination with a carbon-centered organometallic reagent to form the negatively charged sulfinamide intermediate II, we hypothesized that the loss of a phenoxide anion by cleavage of the weak $\mathrm{N}-\mathrm{O}$ bond would be favorable to give the neutral sulfinyl nitrene species III. Upon addition of a second nucleophile such as a further organometallic reagent, or an amine, sulfoximines and sulfonimidamides would be obtained. Importantly, no external oxidant would be needed to achieve the S(VI) oxidation state. Although seemingly straightforward, this reactivity has never previously been achieved. ${ }^{27 \mathrm{~b}}$ This is likely due to difficulties associated with the generation of sulfinyl nitrenes. As noted, sulfinyl azides react with amines via direct substitution to give sulfinamides with loss of $\mathrm{HN}_{3}$ before nitrene formation can occur. ${ }^{29}$ Sulfinyl nitrenes and the related sulfinylnitrenium cation could be formed in the presence of, and reacted with, simple alcohols; ${ }^{27 a}$ however, yields were low and the only sulfur-containing products isolated from this reaction were primary sulfonamides. ${ }^{31}$ Our challenge, therefore, was to develop the first method of generating sulfinyl nitrenes that would allow synthetically useful reactions with common nucleophiles.

We targeted sulfinylamines ${ }^{30}$ derived from $O$-arylhydroxylamines, reasoning that the presence of the aromatic ring would weaken the $\mathrm{N}-\mathrm{O}$ bond and promote the bond cleavage central 


\section{Scheme 5. Mechanistic Observations}
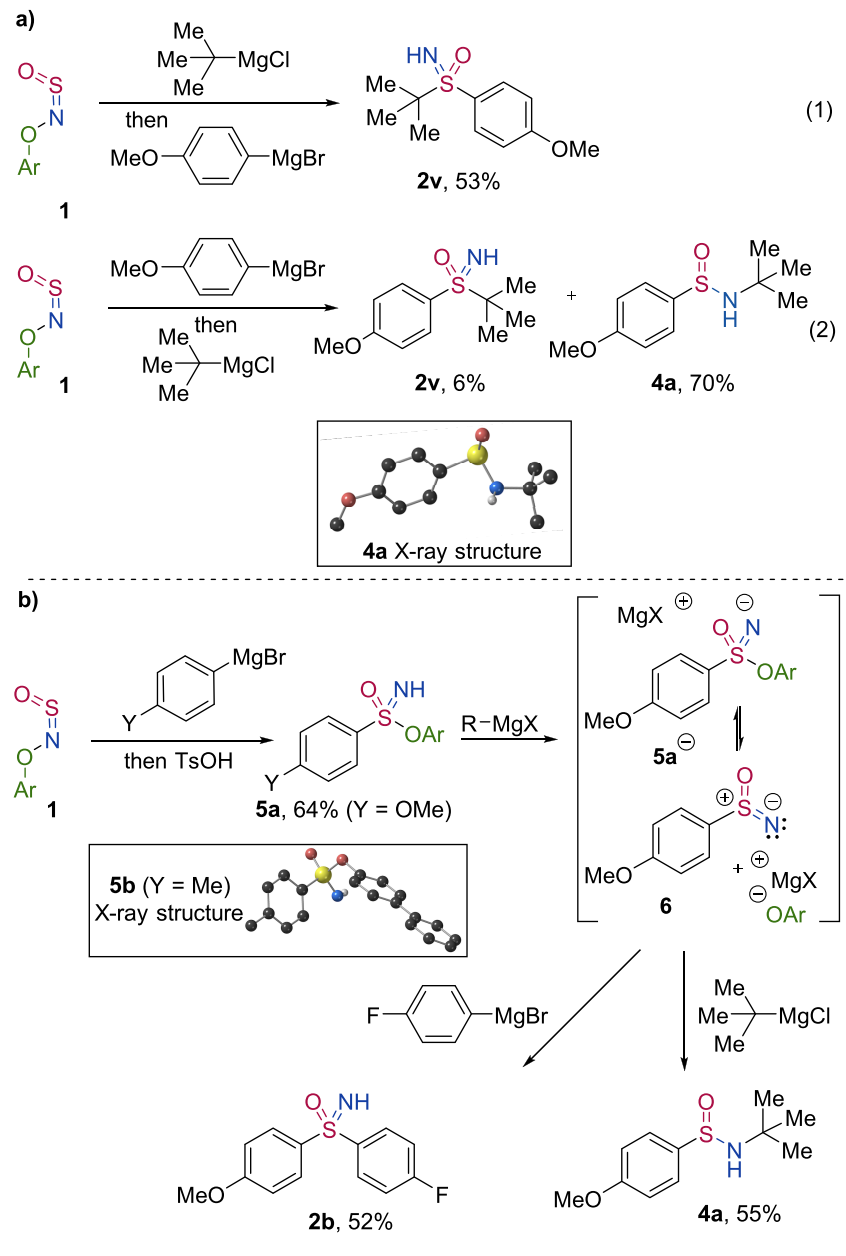

to our reaction design. After some experimentation, we settled on sulfinylhydroxylamine $\mathbf{1}$ (BiPhONSO), a solid reagent that combines good stability with excellent reactivity and can be prepared from biphenylhydroxylamine efficiently on multigram scale (Scheme 4). ${ }^{32}$ Pleasingly, we found that sequential addition of a Grignard reagent and an amine to BiPhONSO at $-78{ }^{\circ} \mathrm{C}$ for $1 \mathrm{~min}$, followed by warming to room temperature, delivered sulfonimidamide $3 \mathrm{a}$ in $85 \%$ yield in accordance with our plan. Crucially, this approach could also be applied to sulfoximines; addition of a second Grignard reagent in place of an amine provided sulfoximine $2 \mathrm{a}$ in $83 \%$ yield. The novel sulfinylamine reagent, $\mathrm{BiPhONSO}$, therefore enables the synthesis of sulfoximines or sulfonimidamides by simple consecutive addition of widely available organometallic reagents and amines in a reaction time of just $15 \mathrm{~min}$.

With the optimized conditions in hand, we set out to determine the scope of sulfoximines that could be prepared (Table 1a). A broad range of aryl Grignard reagents could be used $(\mathbf{2} \mathbf{a}-\mathbf{j})$. The impressive tolerance of the reaction to steric hindrance was shown by the synthesis of a rare ortho,ortho'disubstituted sulfoximine (2h). An organolithium reagent containing an SMe group was also incorporated to obtain a sulfoximine containing mixed-valence sulfur atoms (2i), which could not be prepared using traditional oxidative methods. Likewise, the unprotected basic tertiary amine featured in compound $2 \mathbf{j}$ would be susceptible to oxidation. Medicinally relevant basic $N$-heterocycles such as pyridines and pyrimidines could be incorporated $(\mathbf{2} \mathbf{k}-\mathbf{n})$. Alkyl nucleophiles were also competent in the reaction $(\mathbf{2 o}-\mathbf{w})$. tert-Butylmagnesium chloride could even be used as the initial nucleophile to afford the highly sterically hindered S-tert-butyl sulfoximine $\mathbf{2 v}$ in $53 \%$ yield; such products have previously been prepared by three sequential deprotonations/methylations of an $S$-methyl sulfoximine. ${ }^{33}$ Oxidatively sensitive allyl and vinyl groups were compatible (2w,y). S,S-Dialkyl sulfoximines containing methyl, cyclopropyl, and cyclohexyl groups could also be prepared $(2 \mathrm{z}-\mathrm{ab})$.

The initial product in these syntheses is a sulfoximine anion, and we were able to exploit this by introducing an electrophilic trap as a fourth reaction component (Table 1b). For example, addition of an acid chloride delivered acetyl- and benzoylsubstituted products in high yields (2ac,ad). Urea- and cyanocontaining sulfoximines could also be prepared using a carbamoyl chloride and cyanogen bromide as the electrophile, respectively (2ae,af). An $N$-cyanosulfoximine is incorporated into the marketed insecticide Sulfoxaflor. ${ }^{9}$

We next explored the scope of sulfonimidamides that could be prepared, keeping 4-fluorophenylmagnesium bromide constant as the organometallic component and varying the amine nucleophile (Table 2). The parent 5-, 6-, and 7membered cyclic amines all reacted in high yields $(3 \mathbf{b}-\mathbf{d})$. Cyclic amines bearing an electrophilic ester, acid-sensitive ketal, and oxidatively sensitive thioether functional groups were tolerated $(\mathbf{3 e}-\mathbf{g})$. Bulky noncyclic secondary amines such as dibenzylamine and $\mathrm{N}$-cyclohexylmethylamine worked well $(3 \mathbf{i}, \mathbf{j})$, although a steric limit was reached with diisopropylamine (3k). Primary amines and anilines proved to be competent nucleophiles $(31-p)$. Functionalizations of the antidepressant Amoxapine and amines derived from the antihistamine Loratadine and the antiplatelet medication Clopidogrel were possible $(3 \mathbf{q}-\mathbf{s})$. Amines containing acidic protons, such as alcohols and carbamates, could be used provided that para-toluenesulfonic acid was added prior to the amine $(3 \mathbf{t}, \mathbf{u})$. We then varied the organometallic component, using morpholine or 1-Cbz-piperazine as the amine. Sterically and electronically varied aryl organometallic reagents afforded products in high yields $(3 \mathbf{v}-\mathbf{a a})$. Importantly for medicinal chemists, a range of heteroaryl organometallic reagents performed well, allowing the synthesis of 2- and 3-pyridyl and pyrimidyl sulfonimidamides $(3 \mathbf{a b}-\mathbf{a e})$. Five-membered heterocycles such as thiophene and benzofuran were incorporated in high yields (3af,ag), as was highly basesensitive 4-isoxazole ${ }^{31}$ (3ah), demonstrating the mildness of the reaction conditions. As with the sulfoximines, alkyl Grignard reagents gave good yields of products (3ai-am). The first ever syntheses of $S$-vinyl and $S$-allyl sulfonimidamides were also accomplished (3an,ap). The reliance of previous methods on strongly oxidative conditions and sensitive sulfinyl chloride intermediates may explain why alkenyl substituents were not well-tolerated in the earlier week.

Intriguingly, the formation of $S$-tert-butylsulfoximine $2 \mathbf{v}$ was dependent on the order of addition of the Grignard reagents. When tert-butylmagnesium chloride was added first and 4methoxyphenylmagnesium bromide second, sulfoximine $\mathbf{2 v}$ was isolated in $53 \%$ yield (Scheme $5 \mathrm{a}$, eq 1 ). However, when the order was reversed, $N$-tert-butylsulfinamide $4 \mathbf{a}$ was the major product in $70 \%$ yield and the sulfoximine was present in just $6 \%$ yield (eq 2 ). These results provide strong evidence of a nitrene intermediate; while it is highly improbable that a sulfonimidate ester anion would react with a Grignard reagent on nitrogen, nitrenes are renowned for their electrophilicity at 


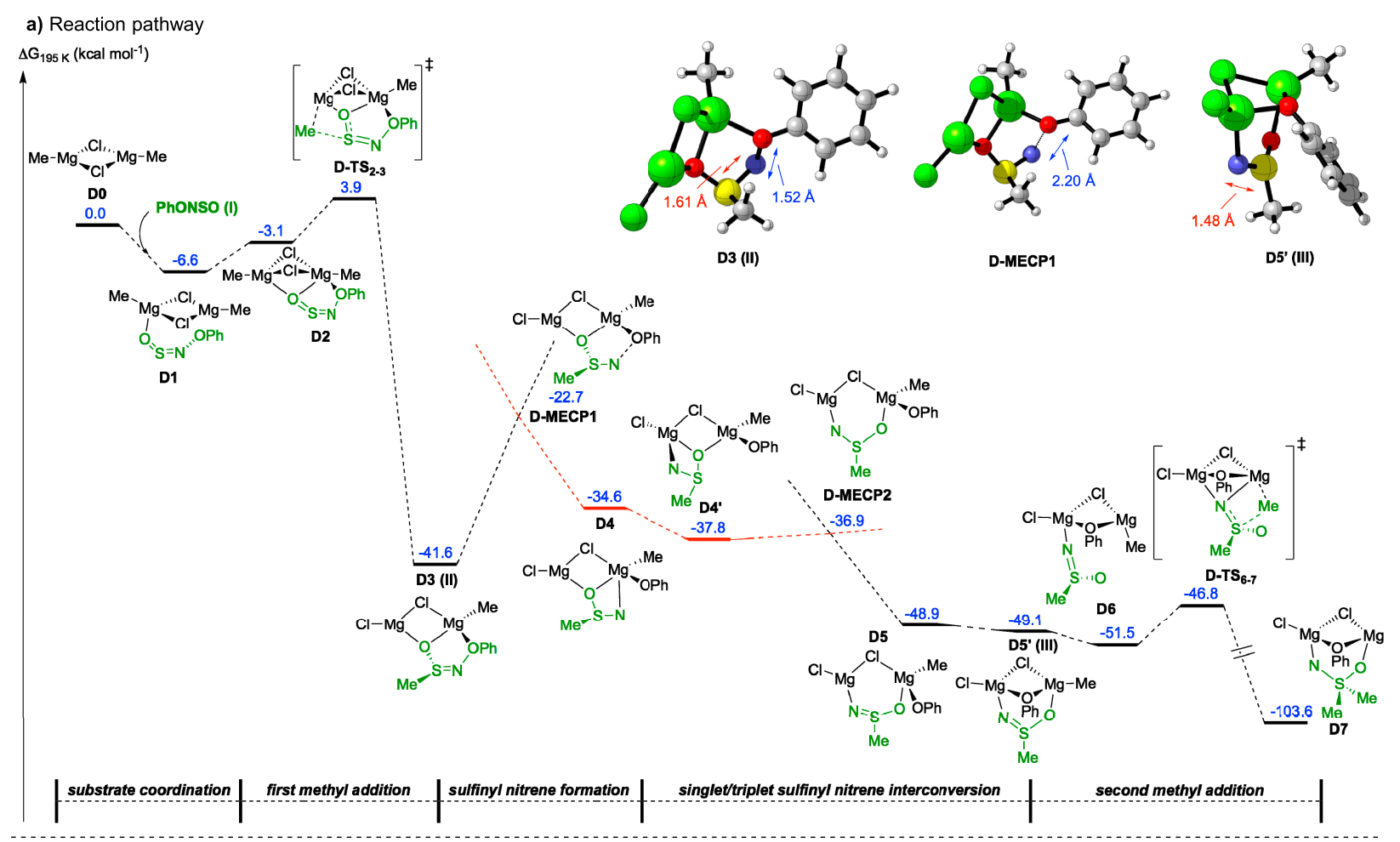

b) S vs N selectivity
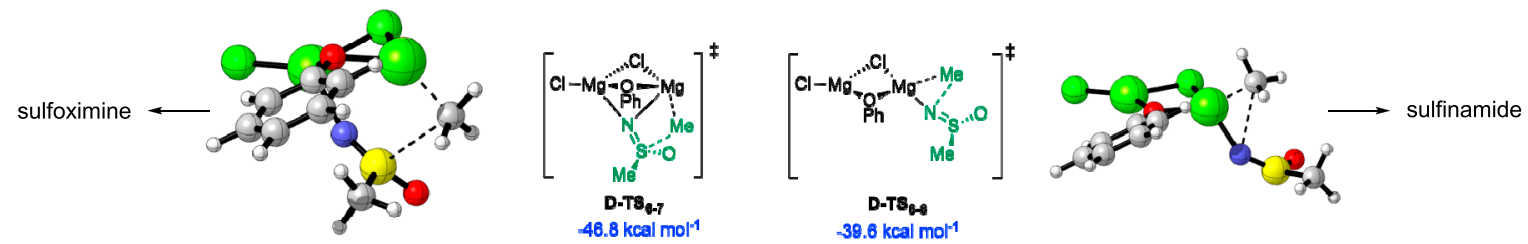

Figure 2. Computational study: (a) Reaction pathway; (b) S vs N selectivity. Energies at the $\operatorname{SMD}(\mathrm{THF})-\omega \mathrm{B} 97 \mathrm{X}-\mathrm{D} / 6-311++\mathrm{g}(\mathrm{d}, \mathrm{p}) / /$ $\operatorname{SMD}(\mathrm{THF})-\mathrm{B} 3 \mathrm{LYP}-\mathrm{BJD} 3 / 6-31 \mathrm{~g}(\mathrm{~d})$ level of theory.

nitrogen. In this case, if the incoming $\mathrm{C}$-nucleophile is sufficiently hindered, attack at the less accessible sulfinyl nitrene sulfur atom could become unfavorable, and formation of the sulfinamide by attack on nitrogen would dominate. We were able to isolate sulfonimidate ester $\mathbf{5 a}$ by addition of paratoluenesulfonic acid at $-78{ }^{\circ} \mathrm{C}$ following addition of the initial Grignard reagent (Scheme $5 b$ ). Reacting sulfonimidate ester 5a with 2 equiv of tert-butylmagnesium chloride resulted also in the formation of sulfinamide $4 \mathbf{a}$. Reaction of $\mathbf{5 a}$ with two equivalents of the aryl Grignard reagent provided sulfoximine $\mathbf{2 b}$ in $52 \%$ yield. Taken together, this suggests that deprotonation of the ester 5a leads to nitrene (6) formation by expulsion of the phenoxide anion. Considering this, it is likely that the anionic sulfonimidate ester $\mathbf{5 \mathbf { a } ^ { - }}$ is formed quickly by recombination of the nitrene (6) and the phenoxide leaving group following $\mathrm{N}-\mathrm{O}$ bond fission, and can act as a source of the nitrene. The related sulfonimidate ester $\mathbf{5 b}$ could also be isolated and yielded X-ray quality crystals; the resultant structure is shown. Experiments using both TEMPO and 1,1-diphenylethylene as radical scavengers in reactions to prepare sulfoximines $\mathbf{2 a}$ and $\mathbf{2 v}$, sulfonimidamide $\mathbf{3 a}$, and sulfinamide $\mathbf{4 a}$, all showed only minimal effect of the additives, suggesting that radical intermediates are likely not involved (see Supporting Information for details).

To gain more insight into the mechanism of this reaction, a computational investigation, employing density functional theory (DFT) methods, was undertaken. ${ }^{28}$ The addition of the Grignard reagent $\mathrm{CH}_{3} \mathrm{MgCl}$ to 1 was investigated, using PhONSO as a model system for sulfinylhydroxylamine $\mathbf{1}$. Figure 2a shows a pathway using a dimeric Grignard species; however, the possibility of an alternative monomeric pathway was also investigated and provided comparable results (see Supporting Information). ${ }^{34}$ The reaction is initiated by formation of the Grignard-substrate complex, D1, which undergoes an irreversible methyl addition through $\mathbf{D}-\mathbf{T S}_{2-3}$ with a barrier of $10.5 \mathrm{kcal} \mathrm{mol}^{-1}$ forming intermediate D3. Subsequently, the anionic sulfinamide component in D3 undergoes intersystem crossing (ISC), from its singlet to a triplet state, forming D4. The barrier of ISC was estimated by locating the minimum energy crossing point (MECP) between the singlet and triplet potential energy surface obtaining DMECP1. D-MECP1 was found to be $19.0 \mathrm{kcal} \mathrm{mol}^{-1}$ higher in energy relative to $\mathrm{D} 3$, having an elongated $\mathrm{N}-\mathrm{O}$ bond $(2.20 \AA$ vs $1.52 \AA$ in D3) while retaining a pyramidal geometry at sulfur. Rotation of the nitrene moiety in D4 to position the nitrogen away from the phenoxide leads to $\mathbf{D 4}^{\prime}$, which then interconverts to the more stable singlet sulfinyl nitrene D5 via D-MECP2 (see Section 2.6 in the Supporting Information). In D5, reorientation of the phenoxide ligand to a bridging position leads to intermediate $\mathbf{D 5}^{\prime}$, a sulfinyl nitrene with a near planar sulfur geometry and a shortened $\mathrm{S}-\mathrm{N}$ bond of 1.48 Å compared to $1.61 \AA$ in D3. Overall, these steps indicate that 
the generation of the sulfinyl nitrene intermediate occurs via a stepwise procedure, with the first ISC involving $\mathrm{N}-\mathrm{O}$ bond fragmentation to a transient triplet intermediate, followed by the second ISC involving the pyramidal sulfinyl nitrene converting to a planar species.

The structural characteristics of the sulfinyl nitrene component in D5' $^{\prime}$ suggest a high degree of electrophilicity at the sulfur center. Indeed, addition of a second methyl group to form sulfoximine $\mathrm{D} 7$ takes place via a low activation barrier $\left(\mathrm{D}-\mathrm{TS} 6-7=4.7 \mathrm{kcal} \mathrm{mol}^{-1}\right)$. Furthermore, the mechanistic experiments indicate the possibility of sulfinamide formation from the nitrene, and hence, this selectivity was also examined (Figure 2b). Comparison of the energies of D-TS $\mathbf{T S}_{-7}$ and D$\mathrm{TS}_{6-8}$ shows the transition state for methyl addition to sulfur $\left(\mathrm{D}-\mathrm{TS}_{6-7}\right)$ to be $7.2 \mathrm{kcal} \mathrm{mol}^{-1}$ more favorable than addition to nitrogen $\left(\mathbf{D}-\mathbf{T S}_{6-8}\right)$, which qualitatively provides the same selectivity as observed experimentally for the majority of examples. Therefore, these results provide a theoretical basis for the reactivity of the sulfinyl nitrene, namely its ability to readily accept a nucleophile at the sulfur center. It is worth noting that in the case of a phenoxide nucleophile, a sulfonimidate ester anion, analogous to $\mathbf{5 a}^{-}$, would be generated reversibly (see Supporting Information, Figure S7). This supports our earlier assertion that this intermediate may act as a nitrene reservoir. Overall, this analysis suggests that formation of sulfoximines (2, D7 in Figure 2a) from sulfinylhydroxylamine (1) is energetically favorable, involving the generation of a key singlet sulfinyl nitrene intermediate with an electrophilic sulfur center.

\section{CONCLUSION}

These results show that sulfinyl nitrenes are available from novel sulfinylhydroxylamine reagents and demonstrate for the first time that they can be combined with carbon- and nitrogen-nucleophiles to provide a diverse range of sulfoximines and sulfonimidamides. We anticipate that this chemistry will transform the preparation of these compound classes, providing a new technology for medicinal and agrochemists to incorporate polar, three-dimensional scaffolds into bioactive molecules. More fundamentally, the ability to conveniently and selectively generate a hitherto virtually unexplored reactive intermediate will catalyze the development of unforeseen chemistry and molecules.

\section{ASSOCIATED CONTENT}

\section{SI Supporting Information}

The Supporting Information is available free of charge at https://pubs.acs.org/doi/10.1021/jacs.0c06986.

Experimental procedures and supporting characterization data and spectra; computational procedures, including Cartesian coordinates (PDF)

Crystallographic data for compound $4 a$ (CIF)

Crystallographic data for compound 2ad (CIF)

Crystallographic data for compound BiPhONSO 1 (CIF)

Crystallographic data for compound $3 \mathbf{y}$ (CIF)

Crystallographic data for compound $\mathbf{5 b}$ (CIF)

\section{AUTHOR INFORMATION}

\section{Corresponding Author}

Michael C. Willis - Department of Chemistry, Chemistry

Research Laboratories, University of Oxford, Oxford OX1 3TA,
U.K.; ๑ orcid.org/0000-0002-0636-6471;

Email: michael.willis@chem.ox.ac.uk

\section{Authors}

Thomas Q. Davies - Department of Chemistry, Chemistry Research Laboratories, University of Oxford, Oxford OX1 3TA, U.K.; $\odot$ orcid.org/0000-0003-1054-1161

Michael J. Tilby - Department of Chemistry, Chemistry Research Laboratories, University of Oxford, Oxford OX1 3TA, U.K.; $\odot$ orcid.org/0000-0002-8916-1454

Jack Ren - Department of Chemistry, Chemistry Research Laboratories, University of Oxford, Oxford OX1 3TA, U.K.

Nicholas A. Parker - Department of Chemistry, Chemistry Research Laboratories, University of Oxford, Oxford OX1 3TA, U.K.

David Skolc - UCB Biopharma SPRL, 1420 Braine-l'Alleud, Belgium

Adrian Hall - UCB Biopharma SPRL, 1420 Braine-l'Alleud, Belgium

Fernanda Duarte - Department of Chemistry, Chemistry Research Laboratories, University of Oxford, Oxford OX1 3TA, U.K.; (1) orcid.org/0000-0002-6062-8209

Complete contact information is available at:

https://pubs.acs.org/10.1021/jacs.0c06986

\section{Notes}

The authors declare no competing financial interest.

\section{ACKNOWLEDGMENTS}

This work was supported by the EPSRC Centre for Doctoral Training in Synthesis for Biology and Medicine (EP/L015838/ 1) and the EPSRC (EP/S03658X/1). Antoine de Gombert and Richard Cooper (both University of Oxford) are thanked for X-ray structure analysis, and the authors acknowledge use of the University of Oxford Advanced Research Computing (ARC) facility in carrying out this work.

\section{REFERENCES}

(1) (a) Scott, K. A.; Njardarson, J. T. Analysis of US FDA-Approved Drugs Containing Sulfur Atoms. Top Curr. Chem. (Cham) 2018, 376, 5. (b) Feng, M.; Tang, B.; Liang, S. H.; Jiang, X. Sulfur Containing Scaffolds in Drugs: Synthesis and Application in Medicinal Chemistry. Curr. Top. Med. Chem. 2016, 16, 1200-1216.

(2) Reggelin, M.; Zur, C. Sulfoximines: Structures, Properties and Synthetic Applications. Synthesis 2000, 2000, 1-64.

(3) Nandi, G. C.; Arvidsson, P. I. Sulfonimidamides: Synthesis and Applications in Preparative Organic Chemistry. Adv. Synth. Catal. 2018, 360, 2976-3001.

(4) Sehgelmeble, F.; Janson, J.; Ray, C.; Rosqvist, S.; Gustavsson, S.; Nilsson, L. I.; Minidis, A.; Holenz, J.; Rotticci, D.; Lundkvist, J.; Arvidsson, P. I. Sulfonimidamides as Sulfonamides Bioisosteres: Rational Evaluation through Synthetic, in Vitro, and in Vivo Studies with $\gamma$-Secretase Inhibitors. ChemMedChem 2012, 7, 396-399.

(5) Frings, M.; Bolm, C.; Blum, A.; Gnamm, C. Sulfoximines from a Medicinal Chemist's Perspective: Physicochemical and in vitro Parameters Relevant for Drug Discovery. Eur. J. Med. Chem. 2017, $126,225-245$.

(6) (a) Lücking, U. Sulfoximines: A Neglected Opportunity in Medicinal Chemistry. Angew. Chem., Int. Ed. 2013, 52, 9399-9408. (b) Chinthakindi, P. K.; Naicker, T.; Thota, N.; Govender, T.; Kruger, H. G.; Arvidsson, P. I. Sulfonimidamides in Medicinal and Agricultural Chemistry. Angew. Chem., Int. Ed. 2017, 56, 4100-4109.

(7) (a) Miller, D.; Thom, S.; St-Galley, S.; Shannon, J.; Leeson, P. Novel Compounds. Patent WO2019/068772A1, 2019. (b) Biftu, T.; 
Khan, T. A. Treating Diabetes with Dipeptidyl Peptidase-IV Inhibitors. Patent WO2014018355A1, 2014.

(8) (a) Lücking, U.; Scholz, A.; Lienau, P.; Siemeister, G.; Kosemund, D.; Bohlmann, R.; Briem, H.; Terebesi, I.; Meyer, K.; Prelle, K.; Denner, K.; Bömer, U.; Schäfer, M.; Eis, K.; Valencia, R.; Ince, S.; Nussbaum, F. v.; Mumberg, D.; Ziegelbauer, K.; Klebl, B.; Choidas, A.; Nussbaumer, P.; Baumann, M.; Schultz-Fademrecht, C.; Rühter, G.; Eickhoff, J.; Brands, M. Identification of Atuveciclib (BAY 1143572), the First Highly Selective, Clinical PTEFb/CDK9 Inhibitor for the Treatment of Cancer. ChemMedChem 2017, 12, 1776-1793. (b) Foote, K. M.; Nissink, J. W. M.; McGuire, T.; Turner, P.; Guichard, S.; Yates, J. W. T.; Lau, A.; Blades, K.; Heathcote, D.; Odedra, R.; Wilkinson, G.; Wilson, Z.; Wood, C. M.; Jewsbury, P. J. Discovery and Characterization of AZD6738, a Potent Inhibitor of Ataxia Telangiectasia Mutated and Rad3 Related (ATR) Kinase with Application as an Anticancer Agent. J. Med. Chem. 2018, 61, 9889-9907. (c) Vendetti, F. P.; Lau, A.; Schamus, S.; Conrads, T. P.; O'Connor, M. J.; Bakkenist, C. J. The orally active and bioavailable ATR kinase inhibitor AZD6738 potentiates the anti-tumor effects of cisplatin to resolve ATM-deficient non-small cell lung cancer in vivo. Oncotarget 2015, 6, 44289-44305. (d) Nishimura, N.; Norman, M. H.; Liu, L.; Yang, K. C.; Ashton, K. S.; Bartberger, M. D.; Chmait, S.; Chen, J.; Cupples, R.; Fotsch, C.; Helmering, J.; Jordan, S. R.; Kunz, R. K.; Pennington, L. D.; Poon, S. F.; Siegmund, A.; Sivits, G.; Lloyd, D. J.; Hale, C.; St Jean, D. J., Jr. Small molecule disruptors of the glucokinase-glucokinase regulatory protein interaction: 3. Structureactivity relationships within the aryl carbinol region of the $\mathrm{N}$ arylsulfonamido-N'-arylpiperazine series. J. Med. Chem. 2014, 57, 3094-3116. (e) Lücking, U.; Jautelat, R.; Krüger, M.; Brumby, T.; Lienau, P.; Schäfer, M.; Briem, H.; Schulze, J.; Hillisch, A.; Reichel, A.; Wengner, A. M.; Siemeister, G. The lab oddity prevails: Discovery of Pan-CDK inhibitor (R)-S-Cyclopropyl-S-(4-\{[4-\{[(1R,2R)-2-hydroxy-1-methylpropyl] oxy\}-5-(trifluoromethyl)pyrimidin-2-yl]amino\}phenyl)sulfoximide (BAY1000394) for the treatment of cancer. ChemMedChem 2013, 8, 1067-1085.

(9) Zhu, Y. M.; Loso, M. R.; Watson, G. B.; Sparks, T. C.; Rogers, R. B.; Huang, J. X.; Gerwick, B. C.; Babcock, J. M.; Kelley, D.; Hegde, V. B.; Nugent, B. M.; Renga, J. M.; Denholm, I.; Gorman, K.; DeBoer, G. J.; Hasler, J.; Meade, T.; Thomas, J. D. Discovery and Characterization of Sulfoxaflor, a Novel Insecticide Targeting Sap-Feeding Pests. J. Agric. Food Chem. 2011, 59, 2950-2957.

(10) (a) Verbelen, B.; Siemes, E.; Ehnbom, A.; Rauber, C.; Rissanen, K.; Wöll, D.; Bolm, C. From One-Pot NH-Sulfoximidations of Thiophene Derivatives to Dithienylethene-Type Photoswitches. Org. Lett. 2019, 21, 4293-4297. (b) Chaabouni, S.; Lohier, J. F.; Barthelemy, A. L.; Glachet, T.; Anselmi, E.; Dagousset, G.; Diter, P.; Pégot, B.; Magnier, E.; Reboul, V. One-Pot Synthesis of Aryl- and Alkyl S-Perfluoroalkylated NH-Sulfoximines from Sulfides. Chem. Eur. J. 2018, 24, 17006-17010. (c) Tota, A.; Zenzola, M.; Chawner, S. J.; St John-Campbell, S.; Carlucci, C.; Romanazzi, G.; Degennaro, L.; Bull, J. A.; Luisi, R. Synthesis of NH-sulfoximines from sulfides by chemoselective one-pot N- and O-transfers. Chem. Commun. 2017, 53, 348-351. (d) Lohier, J.-F.; Glachet, T.; Marzag, H.; Gaumont, A.C.; Reboul, V. Mechanistic investigation of the NH-sulfoximination of sulfide. Evidence for $\lambda^{6}$-sulfanenitrile intermediates. Chem. Commun. 2017, 53, 2064-2067. (e) Bizet, V.; Hendriks, C. M. M.; Bolm, C. Sulfur imidations: access to sulfimides and sulfoximines. Chem. Soc. Rev. 2015, 44, 3378-3390.

(11) (a) Yu, H.; Li, Z.; Bolm, C. Iron(II)-Catalyzed Direct Synthesis of NH Sulfoximines from Sulfoxides. Angew. Chem., Int. Ed. 2018, 57, 324-327. (b) Zenzola, M.; Doran, R.; Degennaro, L.; Luisi, R.; Bull, J. A. Transfer of Electrophilic NH Using Convenient Sources of Ammonia: Direct Synthesis of NH Sulfoximines from Sulfoxides. Angew. Chem., Int. Ed. 2016, 55, 7203-7207. (c) Okamura, H.; Bolm, C. Rhodium-Catalyzed Imination of Sulfoxides and Sulfides: Efficient Preparation of N-Unsubstituted Sulfoximines and Sulfilimines. Org. Lett. 2004, 6, 1305-1307.

(12) Briggs, E. L.; Tota, A.; Colella, M.; Degennaro, L.; Luisi, R.; Bull, J. A. Synthesis of Sulfonimidamides from Sulfenamides via an
Alkoxy-amino- $\lambda^{6}$-sulfanenitrile Intermediate. Angew. Chem., Int. Ed. 2019, 58, 14303-14310.

(13) (a) Mendonça Matos, P.; Lewis, W.; Argent, S. P.; Moore, J. C.; Stockman, R. A. General Method for the Asymmetric Synthesis of NH Sulfoximines via C-S Bond Formation. Org. Lett. 2020, 22, 27762780. (b) Mendonça Matos, P.; Lewis, W.; Moore, J. C.; Stockman, R. A. Sulfonimidates: Useful Synthetic Intermediates for Sulfoximine Synthesis via C-S Bond Formation. Org. Lett. 2018, 20, 3674-3677. (c) Wright, M.; Martínez-Lamenca, C.; Leenaerts, J. E.; Brennan, P. E.; Trabanco, A. A.; Oehlrich, D. Bench-Stable Transfer Reagent Facilitates the Generation of Trifluoromethyl-sulfonimidamides. J. Org. Chem. 2018, 83, 9510-9516.

(14) (a) Greed, S.; Briggs, E. L.; Idiris, F. I. M.; White, A. J. P.; Lücking, U.; Bull, J. A. Synthesis of Highly Enantioenriched Sulfonimidoyl Fluorides and Sulfonimidamides by Stereospecific SuFEx Reaction. Chem. - Eur. J. 2020, DOI: 10.1002/ chem.202002265. (b) Cividino, P.; Verrier, C.; Philouze, C.; Carret, S.; Poisson, J. F. Accessing Enantiopure Endocyclic Sulfoximines Through Catalytic Cycloisomerization of Oxygenated PropargylSulfinamides. Adv. Synth. Catal. 2019, 361, 1236-1240. (c) Aota, Y.; Kano, T.; Maruoka, K. Asymmetric Synthesis of Chiral Sulfoximines through the S-Alkylation of Sulfinamides. Angew. Chem., Int. Ed. 2019, 58, 17661-17665. (d) Aota, Y.; Maeda, Y.; Kano, T.; Maruoka, K. Efficient Synthesis of Cyclic Sulfoximines from N-Propargylsulfinamides through Sulfur-Carbon Bond Formation. Chem. - Eur. J. 2019, 25, 15755-15758. (e) Aota, Y.; Kano, T.; Maruoka, K. Asymmetric Synthesis of Chiral Sulfoximines via the S-Arylation of Sulfinamides. J. Am. Chem. Soc. 2019, 141, 19263-19268. (f) Richards-Taylor, C. S.; Martínez-Lamenca, C.; Leenaerts, J. E.; Trabanco, A. A.; Oehlrich, D. The Synthesis of Trifluoromethyl-sulfonimidamides from Sulfinamides. J. Org. Chem. 2017, 82, 9898-9904. (g) Izzo, F.; Schäfer, M.; Stockman, R.; Lücking, U. A New, Practical One-Pot Synthesis of Unprotected Sulfonimidamides by Transfer of Electrophilic NH to Sulfinamides. Chem. - Eur. J. 2017, 23, 15189-15193. (h) Moragas, T.; Liffey, R. M.; Regentová, D.; Ward, J. P.; Dutton, J.; Lewis, W.; Churcher, I.; Walton, L.; Souto, J. A.; Stockman, R. A. Sigmatropic Rearrangement of Vinyl Aziridines: Expedient Synthesis of Cyclic Sulfoximines from Chiral Sulfinimines. Angew. Chem., Int. Ed. 2016, 55, 10047-10051. (i) Ye, W.; Zhang, L.; Ni, C.; Rong, J.; Hu, J. Stereoselective [3+2] cycloaddition of $\mathrm{N}$-tert-butanesulfinyl imines to arynes facilitated by a removable $\mathrm{PhSO}_{2} \mathrm{CF}_{2}$ group: synthesis and transformation of cyclic sulfoximines. Chem. Commun. 2014, 50, 10596-10599.

(15) Johnson, C. R.; Jonsson, E. U.; Bacon, C. C. Preparation and reactions of sulfonimidoyl chlorides. J. Org. Chem. 1979, 44, 20552061.

(16) For alternative approaches, see: (a) Izzo, F.; Schäfer, M.; Lienau, P.; Ganzer, U.; Stockman, R.; Lücking, U. Exploration of Novel Chemical Space: Synthesis and in vitro Evaluation of NFunctionalized Tertiary Sulfonimidamides. Chem. - Eur. J. 2018, 24, 9295-9304. (b) Dong, S.; Frings, M.; Cheng, H.; Wen, J.; Zhang, D.; Raabe, G.; Bolm, C. Organocatalytic Kinetic Resolution of Sulfoximines. J. Am. Chem. Soc. 2016, 138, 2166-2169. (c) Chen, Y.; Gibson, J. A convenient synthetic route to sulfonimidamides from sulfonamides. RSC Adv. 2015, 5, 4171-4174. (d) Goldberg, F. W.; Kettle, J. G.; Xiong, J.; Lin, D. General synthetic strategies towards Nalkyl sulfoximine building blocks for medicinal chemistry and the use of dimethylsulfoximine as a versatile precursor. Tetrahedron 2014, 70, 6613-6622. (e) Funes Maldonado, M.; Sehgelmeble, F.; Bjarnemark, F.; Svensson, M.; Åhman, J.; Arvidsson, P. I. Synthesis and arylation of unprotected sulfonimidamides. Tetrahedron 2012, 68, 7456-7462.

(17) (a) Mortenson, D. E.; Brighty, G. J.; Plate, L.; Bare, G.; Chen, W.; Li, S.; Wang, H.; Cravatt, B. F.; Forli, S.; Powers, E. T.; Sharpless, K. B.; Wilson, I. A.; Kelly, J. W. "Inverse Drug Discovery" Strategy To Identify Proteins That Are Targeted by Latent Electrophiles As Exemplified by Aryl Fluorosulfates. J. Am. Chem. Soc. 2018, 140, 200210. (b) Dong, J.; Krasnova, L.; Finn, M. G.; Sharpless, K. B. Sulfur(VI) fluoride exchange (SuFEx): another good reaction for click chemistry. Angew. Chem., Int. Ed. 2014, 53, 9430-9448. 
(18) (a) Kitamura, S.; Zheng, Q.; Woehl, J. L.; Solania, A.; Chen, E.; Dillon, N.; Hull, M. V.; Kotaniguchi, M.; Cappiello, J. R.; Kitamura, S.; Nizet, V.; Sharpless, K. B.; Wolan, D. W. Sulfur(VI) Fluoride Exchange (SuFEx)-Enabled High-Throughput Medicinal Chemistry. J. Am. Chem. Soc. 2020, 142, 10899-10904. (b) Smedley, C. J.; Zheng, Q.; Gao, B.; Li, S.; Molino, A.; Duivenvoorden, H. M.; Parker, B. S.; Wilson, D. J. D.; Sharpless, K. B.; Moses, J. E. Bifluoride Ion Mediated SuFEx Trifluoromethylation of Sulfonyl Fluorides and Iminosulfur Oxydifluorides. Angew. Chem., Int. Ed. 2019, 58, 45524556. (c) Liu, F.; Wang, H.; Li, S.; Bare, G. A. L.; Chen, X.; Wang, C.; Moses, J. E.; Wu, P.; Sharpless, K. B. Biocompatible SuFEx Click Chemistry: Thionyl Tetrafluoride $\left(\mathrm{SOF}_{4}\right)$-Derived Connective Hubs for Bioconjugation to DNA and Proteins. Angew. Chem., Int. Ed. 2019, 58, 8029-8033. (d) Gao, B.; Li, S.; Wu, P.; Moses, J. E.; Sharpless, K. B. SuFEx Chemistry of Thionyl Tetrafluoride $\left(\mathrm{SOF}_{4}\right)$ with Organolithium Nucleophiles: Synthesis of Sulfonimidoyl Fluorides, Sulfoximines, Sulfonimidamides, and Sulfonimidates. Angew. Chem., Int. Ed. 2018, 57, 1939-1943. (e) Li, S.; Wu, P.; Moses, J. E.; Sharpless, K. B. Multidimensional SuFEx Click Chemistry: Sequential Sulfur(VI) Fluoride Exchange Connections of Diverse Modules Launched From An $\mathrm{SOF}_{4}$ Hub. Angew. Chem., Int. Ed. 2017, 56, 2903-2908.

(19) Davies, T. Q.; Hall, A.; Willis, M. C. One-Pot, ThreeComponent Sulfonimidamide Synthesis Exploiting the Sulfinylamine Reagent N-Sulfinyltritylamine, TrNSO. Angew. Chem., Int. Ed. 2017, 56, 14937-14941.

(20) For the use of TrNSO in a radical approach to sulfonimidamides, see: Bremerich, M.; Conrads, C. M.; Langletz, T.; Bolm, C. Additions to N-Sulfinylamines as an Approach for the Metal-free Synthesis of Sulfonimidamides: O-Benzotriazolyl Sulfonimidates as Activated Intermediates. Angew. Chem., Int. Ed. 2019, 58, 19014-19020.

(21) McBride, C.; Trzoss, L. L.; Boloor, A.; Sokolo-VA, N.; Pastor, R. M.; Staben, S. T.; Stivala, C.; Volgraf, M.; Bronner, S. M. Sulfonimidamide compounds as inhibitors of interleukin-1 activity. Patent WO2020018975A1, 2020.

(22) Zhang, Z. X.; Davies, T. Q.; Willis, M. C. Modular Sulfondiimine Synthesis Using a Stable Sulfinylamine Reagent. J. Am. Chem. Soc. 2019, 141, 13022-13027.

(23) Johnson, C. R.; Bis, K. G.; Cantillo, J. H.; Meanwell, N. A.; Reinhard, M. F. D.; Zeller, J. R.; Vonk, G. P. Preparation and Reactions of Sulfonimodyl Fluorides. J. Org. Chem. 1983, 48, 1-3.

(24) Johnson, C. R.; Jonsson, E. U.; Wambsgans, A. NucleophilicSubstitution at Sulfur in Sulfonimodyl Compounds - Synthesis of Sulfoximines. J. Org. Chem. 1979, 44, 2061-2065.

(25) For an example showing sulfonimidoyl fluorides reacting with organolithiums to prepare sulfoximines, see ref $18 \mathrm{~d}$.

(26) Gontcharov, A. V.; Liu, H.; Sharpless, K. B. tertButylsulfonamide. A New Nitrogen Source for Catalytic Aminohydroxylation and Aziridination of Olefins. Org. Lett. 1999, 1, 783786.

(27) (a) Maricich, T. J.; Madhusoodanan, S.; Kapfer, C. A. Convergence of Mechanisms Between Sulfinyl azide and NAlkoxysulfinamide Reactions. Tetrahedron Lett. 1977, 18, 983-986. (b) Maricich, T. J.; Hoffman, V. L. Chemistry of Benzenesulfinyl Azides - Reactions with sulfoxides. J. Am. Chem. Soc. 1974, 96, 77707781. (c) Maricich, T. J. Benzenesulfinyl azide and 1,3,5-Triphenyl1,3,5,2,4,6-Tri thiatriazine 1,3,5-Trioxide. J. Am. Chem. Soc. 1968, 90, 7179 .

(28) Wu, Z.; Li, D.; Li, H.; Zhu, B.; Sun, H.; Francisco, J. S.; Zeng, $\mathrm{X}$. Gas-Phase Generation and Decomposition of a Sulfinylnitrene into the Iminyl Radical OSN. Angew. Chem., Int. Ed. 2016, 55, 1507-1510.

(29) Maricich, T. J.; Angeletakis, C. N. Reaction of benzenesulfinyl azide with thiols and amines. Preparation of thiosulfinates and sulfinamides. J. Org. Chem. 1984, 49, 1931-1934.

(30) Kresze, G.; Maschke, A.; Albrecht, R.; Bederke, K.; Patzschke, H. P.; Smalla, H.; Trede, A. Organic N-Sulfinyl Compounds. Angew. Chem., Int. Ed. Engl. 1962, 1, 89-98.
(31) Maricich, T. J.; Jourdenais, R. A.; Albright, T. A. NAlkoxybenzenesulfinamides. Evidence for an alkylation reaction. $J$. Am. Chem. Soc. 1973, 95, 5831-5832.

(32) Appropriate safety precautions should be considered when using hydroxylamine derivatives. See the Supporting Information for DSC data for BiPhONSO. For examples of the process scale use of hydroxylamine-derived reagents, see: (a) Boyles, D. C.; Curran, T. T.; Parlett; Davis, M.; Mauro, F. Electrophilic N-Amination of Two Quinazoline-2,4-diones Using Substituted (Nitrophenyl)hydroxylamines. Org. Process Res. Dev. 2002, 6, 230-233. (b) Shi, Z.; Kiau, S.; Lobben, P.; Hynes, J.; Wu, H.; Parlanti, L.; Discordia, R.; Doubleday, W. W.; Leftheris, K.; Dyckman, A. J.; Wrobleski, S. T.; Dambalas, K.; Tummala, S.; Leung, S.; Lo, E. Development of a Practical Synthesis of a p38 Kinase Inhibitor via a Safe and Robust Amination. Org. Process Res. Dev. 2012, 16, 1618-1625.

(33) Levacher, V.; Eriksen, B. L.; Begtrup, M.; Dupas, G.; Quéguiner, G.; Duflos, J.; Bourguignon, J. The tert-butyl sulfoximine group as an effective ortho-director of lithiation: Ortho-metallated S(tert-butyl)-S-phenylsulfoximines. Tetrahedron Lett. 1999, 40, 16651668.

(34) Peltzer, R. M.; Gauss, J.; Eisenstein, O.; Cascella, M. The Grignard Reaction - Unraveling a Chemical Puzzle. J. Am. Chem. Soc. 2020, 142, 2984-2994. 